\title{
Comment on "Ring-breaking electron attachment to uracil: Following bond dissociations via evolving resonances" [J. Chem. Phys. 128, 174302 (2008)]
}

\author{
Carl Winstead ${ }^{\text {a) }}$ and Vincent McKoy \\ A. A. Noyes Laboratory of Chemical Physics, California Institute of Technology, Pasadena, \\ California 91125, USA
}

(Received 14 May 2008; accepted 21 July 2008; published online 15 August 2008)

[DOI: $10.1063 / 1.2969818]$

Because low-energy resonances play a critical role in electron-driven dissociation of nucleobases, their assignment is fundamental to an understanding of the dissociation dynamics. In a 2004 paper, ${ }^{1}$ Gianturco and Lucchese reported energies and widths for electron scattering resonances in uracil, claiming good agreement between their computed energies for the $\pi^{*}$ resonances and experimental values. ${ }^{2}$ In a subsequent Comment, ${ }^{3}$ Burrow pointed out that the claimed agreement depended on misidentifying the first and second calculated $\pi^{*}$ resonances with, respectively, the second and third experimental resonances. A recent paper by Gianturco et $a .^{4}$ reports new calculations for uracil employing substantially the same scattering model and again claims close agreement with experimental $\pi^{*}$ resonance positions, and also with those of other calculations. ${ }^{5,6}$ Although the resonance positions that Gianturco et al. obtain (1.7, 3.5, and $6.5 \mathrm{eV}$ ) (Ref. 4) are, in fact, substantially the same as in their earlier work $(2.27,3.51$, and $6.50 \mathrm{eV}){ }^{1}$ they point to a new feature at $0.33 \mathrm{eV}$ as the first $\pi^{*}$ resonance, thereby justifying the identification of the three higher-energy peaks as second, third, and fourth $\pi^{*}$ resonances. They ascribe the appearance of this new feature to the use of a larger partialwave expansion. ${ }^{4}$

The purpose of the present Comment is to point out that the calculated $0.33 \mathrm{eV}$ peak cannot be the first $\pi^{*}$ resonance; rather, it is likely a computational artifact of the strong dipole potential of uracil, of a kind already seen in earlier work. ${ }^{6}$ The more recent calculations of Gianturco et al. thus continue to place the $\pi^{*}$ resonances too high in energy. Several lines of evidence compel this conclusion:

(1) On chemical grounds, one expects not four but three $\pi^{*}$ shape resonances in uracil, corresponding to the three empty $\pi$ valence orbitals. Other scattering calculations ${ }^{5,6}$ indeed produce only three.

(2) The $\pi^{*}$ resonances of uracil are centered on the ring and analogous to the $\pi^{*}$ resonances of benzene: In addition to the node in the plane of the ring, the first and second resonances, $\pi_{1}^{*}$ and $\pi_{2}^{*}$, have two azimuthal nodes, while the third resonance, $\pi_{3}^{*}$, has three azimuthal nodes. As already remarked by Burrow, ${ }^{3}$ Figs. 7 and 8 of Ref. 1, showing the nodal patterns associated with the resonant states, conclusively support the identification of the calculated features at 2.27, 3.51, and $6.50 \mathrm{eV}$ as $\pi_{1}^{*}, \pi_{2}^{*}$, and $\pi_{3}^{*}$, respectively. This assign- ment points to the $1.7 \mathrm{eV}$ feature in the later calculation, ${ }^{4}$ and excludes the $0.33 \mathrm{eV}$ feature, as $\pi_{1}^{*}$.

(3) Because $\pi_{1}^{*}$ has a simple nodal structure_as simple as that of $\pi_{2}^{*}$, and simpler than that of $\pi_{3}^{*}$-there is no physical reason to expect that one would need to carry the calculation to higher partial waves to obtain it.

(4) On the other hand, high partial waves are needed to approximate well the long-range scattering mediated by uracil's static dipole moment. By the same token, when $\mathcal{L}^{2}$ scattering methods are used, spatially extended basis sets are required. Earlier work on uracil ${ }^{6}$ showed that, in the ${ }^{2} A^{\prime \prime}$ representation where the $\pi^{*}$ resonances occur, the principal difference between a small basis set and larger, extended basis sets is that the latter produce a strong rise in the cross section as the energy decreases toward $0 \mathrm{eV}$, while the former does not; at higher energies, both large and small basis sets yield the three $\pi^{*}$ resonances at nearly the same positions (see Figs. 1 and 2 of Ref. 6). One would expect a similar dipolar enhancement of the low-energy cross section in oneelectron calculations carried to high partial waves, such as those of Ref. 4. Very near $0 \mathrm{eV}$, the dipolar rise in the ${ }^{2} A^{\prime \prime}$ cross section may turn over, due to the finite size of the calculation, producing an artifactual peak. Such behavior was seen in Ref. 6. The $0.33 \mathrm{eV}$ peak identified by Gianturco et $a l .{ }^{4}$ as the $\pi_{1}^{*}$ resonance appears to be the same sort of artifact. One may note, in particular, the strong qualitative resemblance between their ${ }^{2} A^{\prime \prime}$ cross section (Ref. 4, Fig. 2, bottom) and earlier calculations at the static-exchange level (Ref. 6, Fig. 1, bottom).

(5) Finally, as discussed elsewhere, ${ }^{7}$ the correlation/ polarization dynamics determining the position of $\pi_{3}^{*}$ appear to be fundamentally different from those involved in $\pi_{1}^{*}$ and $\pi_{2}^{*}$ and to involve explicit channel coupling, making it unlikely that a one-electron scattering model could simultaneously produce excellent energies ( $0.3 \mathrm{eV}$ maximum error) for all three. Indeed, such a one-electron model applied to benzene yields a fair position for $\pi_{1}^{*} / \pi_{2}^{*}(0.7 \mathrm{eV}$ error $)$ but a $\pi_{3}^{*}$ energy that is considerably poorer $(2.6 \mathrm{eV}$ error $){ }^{8}$

Considering the large errors in the $\pi^{*}$ resonance energies at the equilibrium geometry, great caution clearly must be exercised in assessing the results of Ref. 4 for $\pi^{*}$ and $\sigma^{*}$ 
resonance energies as functions of nuclear geometry, including certainly the locations if not also the occurrence of conical intersections among them.

${ }^{a)}$ Electronic mail: carl@schwinger.caltech.edu.

${ }^{1}$ F. A. Gianturco and R. R. Lucchese, J. Chem. Phys. 120, 7446 (2004).

${ }^{2}$ K. Aflatooni, G. A. Gallup, and P. D. Burrow, J. Phys. Chem. A 102,
6205 (1998).

${ }^{3}$ P. D. Burrow, J. Chem. Phys. 122, 087105 (2005).

${ }^{4}$ F. A. Gianturco, F. Sebastianelli, R. R. Lucchese, I. Baccarelli, and N. Sanna, J. Chem. Phys. 128, 174302 (2008).

${ }^{5}$ S. Tonzani and C. H. Greene, J. Chem. Phys. 124, 054312 (2006).

${ }^{6}$ C. Winstead and V. McKoy, J. Chem. Phys. 125, 174304 (2006).

${ }^{7}$ C. Winstead and V. McKoy, Phys. Rev. Lett. 98, 113201 (2007).

${ }^{8}$ F. A. Gianturco and R. R. Lucchese, J. Chem. Phys. 108, 6144 (1998). 